\title{
Electrostatically charged COVID-19 disinfectant spray coverage on a cylinder
}

\author{
K. M. Bade*, J. A. Swift, P. W. Vesely, and R. J. Schick \\ Spray Analysis and Research Services, Spraying System Co., USA \\ ${ }^{*}$ Corresponding author email: kyle.bade@spray.com
}

\begin{abstract}
Surface disinfecting has become a necessity in the global effort to control the COVID-19 pandemic, and the application of disinfectant liquid to completely and uniformly cover target objects is a common goal. Spraying of these materials offers a clearly beneficial solution where the liquid is atomized into droplets which are distributed on the target surface. However, while surfaces in the direct line-of-sight may receive adequate coverage, hidden surfaces on the side and back of an object may be shielded and receive limited or no coating. Electrostatically charged sprays have received added attention and use because, in concept, a charged droplet may be more likely to not only land on a surface through direct impingement, but also be drawn further toward hidden surfaces due to attractive charge forces. Therefore, electrostatically charged sprayers provide a uniquely beneficial method to distribute disinfectant materials, although the actual effects have received limited scientific analysis. In the present study, a commercially available electrostatic sprayer has been investigated with electrostatic spray charge off and on in order to evaluate the resulting coating completeness, uniformity, and efficiency. Measurements of the spray drop size distribution upstream of the target cylinder offer insight for differences in the resulting coverage. While this investigation is not comprehensive, it serves to offer insight into the potential benefits of electrostatically charged disinfecting sprays as well as explanations into how those improvements are achieved. Using the present sprayer, disinfectant material, and target object, coverage was improved by $40 \%$ using charged droplets which was primarily due to increased coverage on the downstream (back) side of the target cylinder, which saw zero coverage without charged droplets.
\end{abstract}

\section{Keywords}

Electrostatically Charged Spray, Coating, Disinfectant, COVID-19, Experimental

\section{Introduction}

Electrostatic sprayers and nozzles have been researched and manufactured for several decades for various applications including agricultural [1], lubrication [2] [3], and combustion [4], among other applications. Electrostatic sprayers are now a popular choice for disinfectant formulation spraying with the desire to spray coat all surfaces of complex items like hand rails, chairs, desks, door knobs, etc. The spray produced by a traditional nozzle impacts the surfaces it comes directly in contact with but potentially excludes hard to reach surfaces leaving them untreated (ex: underside of a hand rail). A charged spray plume is attracted to neutral and electrically grounded surfaces improving the possibility of electrostatic forces acting on droplets influencing their trajectory from movement based purely on momentum, gravity, or air currents entrainment.

As of mid-March 2021, the Johns Hopkins University of Medicine Coronavirus Resource Center [5] reports that the SARS-CoV-2 virus has caused over 120 million infections and 2.6 million fatalities worldwide. Even with recent approval of a vaccines, the world's population will be fighting to avoid infection from this virus for years as efforts are made to achieve heard immunity through vaccination for the roughly 7.8 billion people on this planet. Spraying of disinfectants is one of several important tools in preventing the spread of this virus in public spaces such as schools, offices, and medical facilities; as well as future disinfecting needs in order prevent infectious public spaces and surfaces. 
Spray coating of cylindrical objects is typically achieved by rotating the sprayer or cylinder during the coating process. However, when applying a disinfectant, the sprayer is typically handheld, with the goal of processing a room or area quickly, in which case careful attention is not given to individual objects, but to an area. In this sense, individual objects may only receive a momentary spray from a nominally stationary and singular source; this is the premise of the current experiments with a single stationary nozzle spraying onto a vertically positioned cylinder. Coating of the cylinder through direct impact may apply the spray material to the front surface only [6] [7], and will depend greatly on air/droplet path around the cylinder and resulting recirculation pattern on the rear of the cylinder. Many experiments have been conducted looking at thin-wire coating [8], however, the this presents a very different flow path where the passing airflow Reynolds number $(\mathrm{Re})$ may be $<40$ allowing for laminar, or only small scale recirculation albeit sufficient to coat the wires. For larger cylinders, with $R e>40$, an alternating shedding pattern will form and carry droplets downstream [9] [10]. Depending on the droplet size, the air currents may recirculate small low-momentum droplets, but it is found that medium to large droplets often cross streamlines and flow beyond the cylinder without even being drawn near the rear of the cylinder. It is in this scenario that electrostatic charge applied to the droplets offers an additional body force which may act to draw these droplets closer to the cylinder and increase coverage and deposition and is the premise of the presented work.

\section{Experimental Methods and Setup Overall Setup: Nozzle, Spray Material, Cylinder}

The experimental setup for this study utilized a commercial electrostatic sprayer, a disinfectant liquid proven to kill the COVID-19 virus, and a metallic cylinder positioned vertically to be sprayed with the disinfectant. Tests were conducted with and without an electrostatic charge applied to the disinfectant material, and the coverage and spray characteristics on/near the cylinder were investigated to explore the coating quality and uniformity.

A Ryobi ${ }^{\circledR} 18 \mathrm{~V}$ ONE+ cordless 1 gallon electrostatic sprayer operated at the medium spray setting was used for all experiments. This spray system utilizes a Spraying Systems Co.® UniJet@ hydraulic full cone nozzle, part number TG-0.4, providing a flow rate of $0.36 \mathrm{lpm}$, which indicates that the nozzle was operating at 4.5 bar with the Ryobi system. The spray charge used in this system imparts a positive charge to the spray fluid, which is discussed in more detail in the Spray Specific Charge Measurement section.

For all tests, the PathoSans ${ }^{\circledR}$ on-site generated, PathoCide ${ }^{\circledR}$ sanitizer and disinfectant material was used as the spray medium which has effectively the same density, viscosity, and surface tension as water. PathoCide material is a hypochlorous acid solution composed of softened water and 165-200 ppm of Hypochlorous Acid (0.0165-0.02\%); PathoCide contains no bleach, phosphates, ammonia, or petrochemicals, some of which may be found in other disinfectant materials. The on-site device-produced PathoCide solution has been proven to kill the SARS-CoV-2 virus causing the COVID-19 pandemic, by a third party GLP accredited lab, making it an ideal candidate material for these disinfectant spray investigations.

The target object for coating in these tests was a cylinders of $88.9 \mathrm{~mm}$ diameter, 304.8 $\mathrm{mm}$ tall, made of 6061 aluminum (metal). Figure 1 provides an overall view of the nozzle and cylinder arrangement with a global coordinate axis which is defined to be located at the centerline height of the nozzle on the front-face of the cylinder. The nozzle was positioned at $(x, y, z)=(0,-304.8,0) \mathrm{mm}$. Additionally, Figure 1 demonstrates the location of the various test type regions/locations showing the Water Sensitive Paper (WSP - light gray regions) and Free Available Chlorine (FAC - small blue squares) on the cylinder surface. The sprayer and nozzle were connected using a common rail along the lower edge of the cylinder, which allowed the sprayer and cylinder to be traversed together for alignment during PDI measurements. 


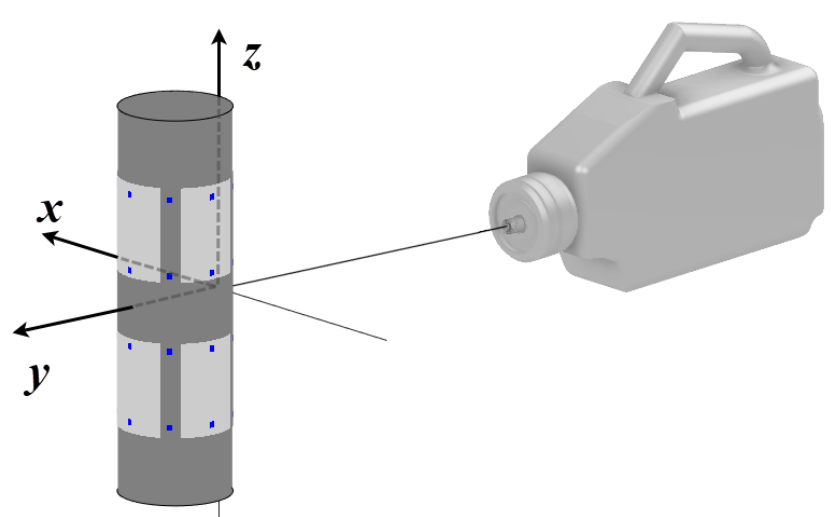

Figure 1. Setup schematic of the sprayer and nozzle, target cylinder, coordinate axis, and measurement locations

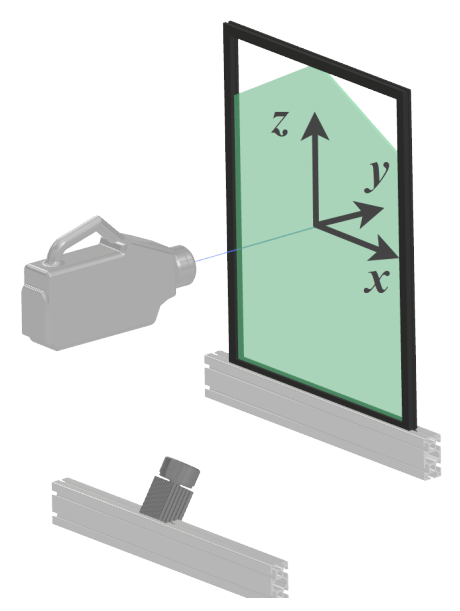

Figure 2. SprayScan mPT setup and image plane

\section{Spray Specific Charge Measurement}

To validate that the spray plume was charged, an experiment was conducted to measure the spray specific charge. A faraday cup experiment was conducted by spraying into a conductive metal pan that was electrically insulated from its environment and was connected to earth ground via a wire and grounding rod. A Keithley 6485 picoammeter was used to measure the current passing from the metal pan to earth ground. This instrument can measure current from $20 \mathrm{fA}$ to $20 \mathrm{~mA}$ with $10 \mathrm{fA}$ resolution at up to $1 \mathrm{kHz}$ sampling rate [11]. The mass flow rate of the spray was determined by measuring the weight change in the supply tank of the sprayer over time. This spray current measurement technique has been used in previous electrostatic spray studies [12] [13] and the methods are described in more detail by Yule et al. [12]. Spray specific charge, or charge to mass ratio, is the ratio of spray current (ampere) and mass flow rate (grams per second) resulting in units of Coulomb per kilogram $(C / \mathrm{kg})$.

\section{Water Sensitive Paper (WSP)}

Water Sensitive Paper (WSP) is a thin, flat, rigid paper coated with a bromothymol blue stain, which turns from yellow-to-blue when it encounters aqueous droplets. WSP is typically used for the qualitative evaluation of where droplets land on a target surface; however, by imaging stained paper, the coverage was quantified. More information on these WSP cards and the methods are described by Hoffman and Hewitt [14], and these papers were provided by TeeJet, Spraying Systems Co., or Syngenta. All results are binary 1/0 (blue/yellow) indicating the presence or absence of spray reaching the surface, but in the case of the current effort, this provided a very direct and indicative technique for evaluating the overall coverage on the target object. WSP is effective in environments with up to $80 \%$ humidity, although exposure to high humidity environments will activate the paper, so limiting exposure outside of the testing time is critical. Droplets of $D=50 \mu \mathrm{m}$ and larger reliably mark the WSP, and smaller droplets will still provide an ensemble effect. Digital images of the WSP were acquired after spraying to allow the percent coverage on each surface to be evaluated and mapped and the blue/yellow areas were converted to black/gray, respectively, as can be seen in Figure 3. This method will only show areas of each surface that have been covered; it will not show the distribution variations throughout the spray area. Figure $3 a$ demonstrates the placement of the WSP rectangular areas with the black/gray regions on the front, back, left, and right of the target cylinder, at two $z$ positions, as a function of the angle around the cylinder (with $\theta=0^{\circ}$ at the center of the front face, in-line with the nozzle). 


\section{Free Available Chlorine (FAC)}

Free Available Chlorine (FAC) is the concentration of chlorine within a disinfectant that can sanitize a surface. For testing, chlorine sensitive testing strips were used to identify how much chlorine was deposited onto various surface regions. Multiple strips were placed directly on the substrates to assess the ability of each spray to evenly apply the disinfectant. When sprayed with PathoCide® Sanitizer and Disinfectant, the strips directly measure the FAC concentration; measured in parts per million (PPM) with a maximum concentration of $310 \mathrm{ppm}$. Evaluating the FAC concentration, specifically around a 3D substrate, allows verification on whether there were appropriate surface contact levels of chlorine without spraying directly on a surface.

The FAC test is separate from the WSP test. Where the WSP indicates the spray coverage over a wide area of the substrate, the FAC strips are smaller and only detect the chlorine concentration at a few distinct locations.

\section{Laser Sheet Imaging}

Planar imaging of the spray was conducted upstream of the cylinder to evaluate the size, shape, and distribution of the spray plume before interacting with the cylinder. A laser sheet imaging system from Spraying Systems, the SprayScan $\AA \mathrm{mPT}$ system, was used and the setup may be seen in Figure 2. A $50 \mathrm{~mW}, 532 \mathrm{~nm}$ wavelength laser beam is passed through a diverging lens to create a $110^{\circ}$ diverging laser sheet, approximately $1 \mathrm{~mm}$ thick. The laser sheet is positioned normal to the primary spray direction. As the spray passes through the laser sheet, the cross section of the plume is illuminated and imaged. A series of approximately 200 images was collected. The image intensity field is a product of the scattered laser light intensity (Mie theory) and is therefore proportional to the distributed surface area of the passing spray; a product of droplet size and count (i.e. higher intensity regions may be attributed to large droplets and/or high ensemble droplet concentration).

\section{Laser Diffraction}

A Sympatec HELOS/KR-VARIO laser diffraction instrument with the PAQXOS v3.0 software was used for drop size measurements of the spray at a spray distance of $304.8 \mathrm{~mm}$ to provide an overall assessment of the drop size range that will interact with the cylinder and potentially deposit on its surface. The PAQXOS 3.0 software uses the Fraunhofer Enhanced Evaluation (FREE) calculation method, based on the Fraunhofer diffraction theory, to evaluate the laser diffraction signals and determine the drop size distribution. The laser diffraction instrument was operated with the R6 lens, providing a laser diameter of $26 \mathrm{~mm}$, maximum measurement distance of $566 \mathrm{~mm}$, and a measurable drop size range of $0.5-1750 \mu \mathrm{m}$ within 31 non-linearly spaced bins; with all droplets found to be $\leq 550 \mu \mathrm{m}$ this primarily used the lower 25 bins.

Testing was conducted by positioning the electrostatic nozzle so that the spray plume was well above the measurement region, spraying horizontally with no spray falling in to the measurement region, operating the sprayer at a steady condition, and then traversing the sprayer vertically downward at a constant speed of approximately $0.075 \mathrm{~m} / \mathrm{s}$. This vertical movement was very slow with respect to the spray velocity, resulting in a negligible effect. Drop size measurements were acquired during the entire nozzle traverse time, resulting in a single, overall assessment of the drop size distribution across the entire spray pattern.

\section{Results and Discussion Cylinder Surface Coverage}

To begin the investigation, WSP was placed on the surface of the cylinder at eight locations covering the front, back, left, and right sides at two heights. Figure 3 demonstrates the cylinder surface area as a function of angle around the cylinder and height relative to the height of nozzle $(\theta, z)$. Where $\theta$ represents the angle around the cylinder with 0 degrees at the front-center and 180 degrees at the back-center, and $z$ representing the heights relative to height of the 


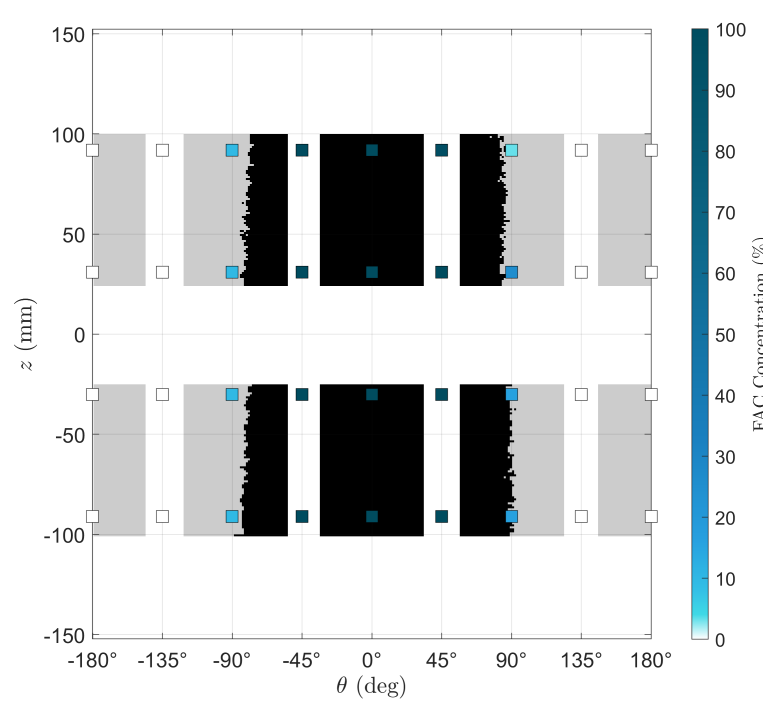

(a) Electrostatics off (ES off $)$

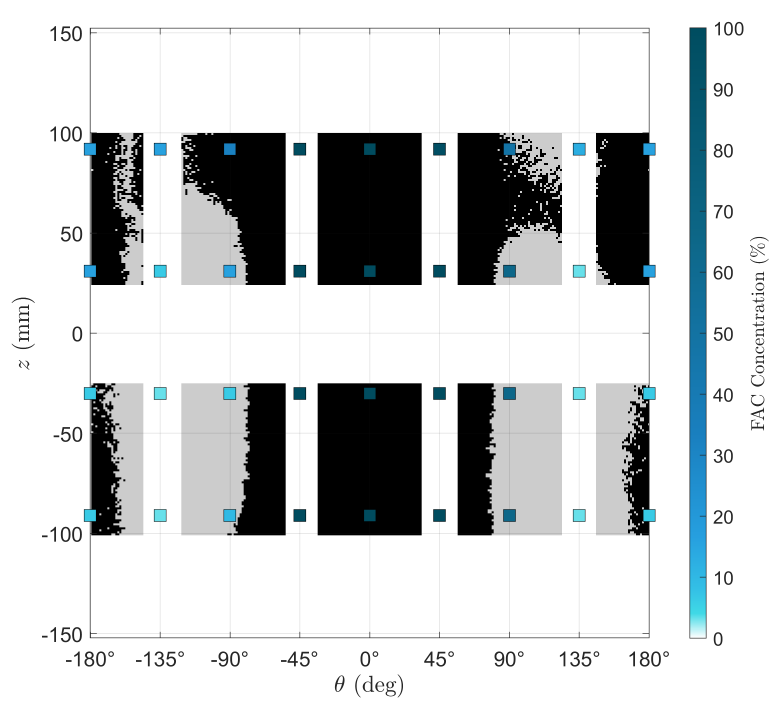

(b) Electrostatics on (ES on

Figure 3. WSP and FAC results with the metallic cylinder and the nozzle at $304.8 \mathrm{~mm}$ spray distance

nozzle. The WSP areas are shown with black/gray regions representing the blue/yellow WSP areas after spraying, respectively; the blue-squares and colorbar represent the FAC results and will be discussed after the WSP results. In Figure $3 a$, the coverage result is provided using the metallic cylinder with the nozzle at a $304.8 \mathrm{~mm}$ spray distance $(y=304.8 \mathrm{~mm})$ and the electrostatics turned off $\left(E S_{\text {off }}\right)$, and demonstrates the spray directly impacting the front face from approximately, $-75^{\circ}<\theta<90^{\circ}$. The coverage on the front face is slightly wider at the lower end $(-z)$ of the cylinder with a small bias toward the positive $\theta$ side which would indicate a stagnation point at approximately $\theta=+5^{\circ}$.

In Figure $3 \mathrm{~b}$, the electrostatics are turned on $\left(\mathrm{ES}_{\mathrm{on}}\right)$ and an increase in coverage on the back and back-sides is immediately observed. While the $\mathrm{ES}_{\text {off }}$ case saw no deposition on the back-half of the cylinder, the $\mathrm{ES}_{\text {on }}$ case saw at least some deposition over the entire $z$ range of the cylinder. Furthermore, the back-side coverage is more complete (not speckled) in the $+z$ region, which is above the nozzle height. This is due to the larger droplets preferentially falling to the lower-half due to gravity as the spray approaches the cylinder, while the smaller droplets remain higher and are then attracted toward the back of the cylinder moreso on the top-half.

Overall, the WSP covered $31,616 \mathrm{~mm}^{2}$ on the surface of the cylinder. With ES $\mathrm{off}_{\text {, the spray }}$ coverage area was found to to $14,622 \mathrm{~mm}^{2}$, or $46 \%$ of the WSP covered cylinder's surface. However, with $\mathrm{ES}_{\text {on }}$, the coverage area was increased to $20,471 \mathrm{~mm}^{2}$, which was $65 \%$ of the WSP paper area. Therefore, by adding the electrostatic charge to the spray, the coverage area was increased by $40 \%$, a significant change.

The WSP results provide an overall perspective on the liquid deposition regions, however, this very simple $1 / 0$ result does not allow differentiation about exactly how much material was deposited over cylinder area. In order to assess a quantitative distribution of where on the cylinder more, or less, material was deposited, FAC strips were positioned near the top and bottom edge of each WSP region, centered in $\theta$. Figures $3 a$ and $3 b$ demonstrate these FAC strip locations with small blue squares which are drawn to scale on the cylinder and within the WSP regions. The blue color of the FAC strips indicates the mass of chlorine reaching at each location. Figure 4 provides the FAC results, at each height of the cylinder, over the theta locations. A reminder, the digitally imaged FAC strips were assessed across a range of 20 shades of blue, providing a $\pm 2.5 \%$ binning of the results (for an estimated $5 \%$ accuracy).

Reviewing the FAC results shown in Figure 4, with the electrostatics turned on an increase in deposition on the top-half of the cylinder is found on the sides \& back, while the lower-half sees 


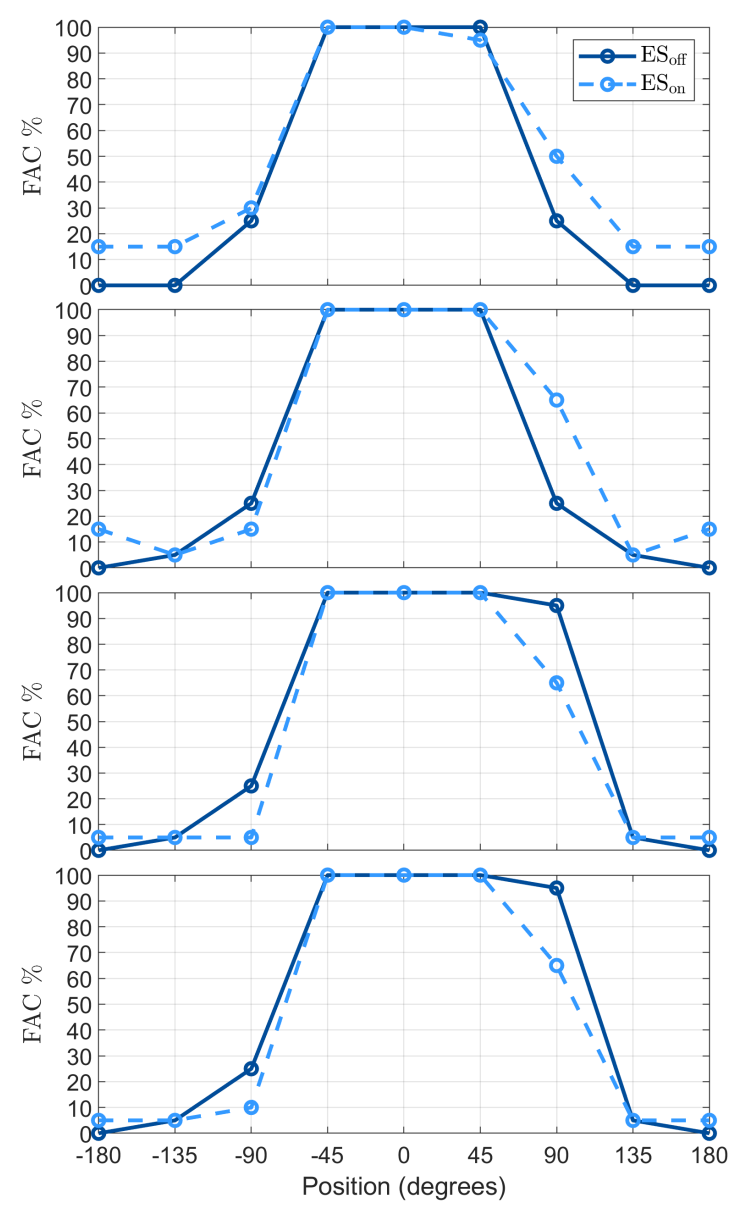

Figure 4. FAC concentration results for the metallic cylinder at $304.8 \mathrm{~mm}$ spray distance with the electrostatic charge on and off. The bottom-to-top figures are provided at heights of $z=-90,-30,30$, and $90 \mathrm{~mm}$ respectively.

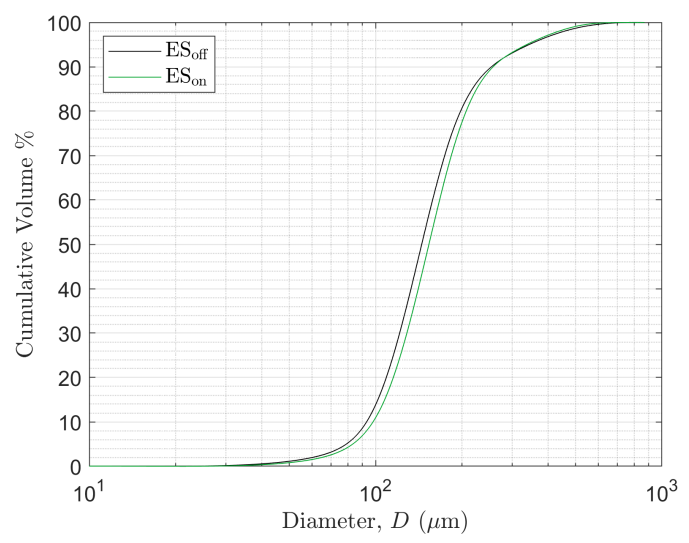

Figure 5. Laser diffraction drop size testing results for $\mathrm{ES}_{\text {off }}$ and $\mathrm{ES}_{\text {on }}$ at $z=304.8 \mathrm{~mm}$

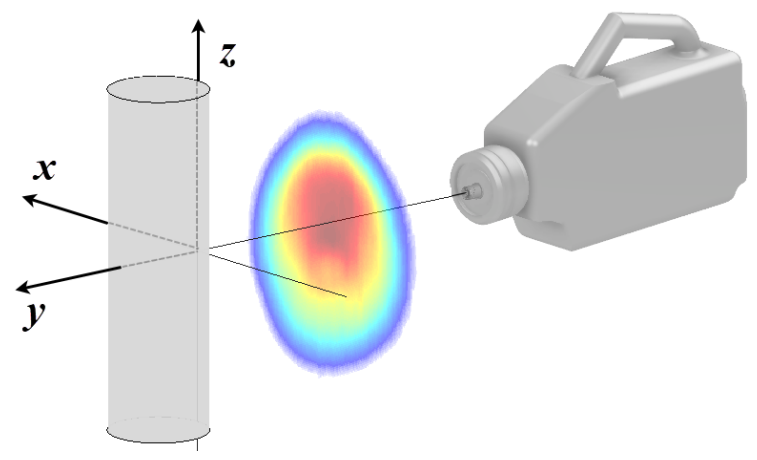

Figure 6. 2D Spray distribution upstream of the cylinder at $152.4 \mathrm{~mm}$ from the cylinder

a reduced deposition on the sides and a slight increase from zero at the back-middle. Looking first at the lower-half of the metallic cylinder, the FAC results are consistent with the WSP results of Figure 3 which demonstrate this same pattern of separating from the cylinder upstream of $\theta< \pm 90^{\circ}$, and redepositing on the back-middle between $\theta= \pm 165^{\circ}$ the electrostatic charge has caused droplets to deposit on the back-middle surface. For the top-side of the metallic cylinder, Figure 4, the coverage experiences the greatest and more uniform increase in deposition for all the tested cases increasing from zero back-side coverage with no electrostatics, up to about $15 \%$ deposition on both sides.

In order to better understand why an increased back-side deposition was observed with the electrostatic charge, the next sections will explore the upstream spray characteristics in more detail as well as the spray charge details.

\section{Spray Characteristics}

In order to understand the spray characteristics upstream of the cylinder, 2D spray distribution/pattern (laser sheet imaging) testing was conducted $152.4 \mathrm{~mm}$ from the nozzle exit orifice $(y=-152.4 \mathrm{~mm})$ in the $x z$-plane, to assess the spray at this cross-section. Additionally, laser diffraction measurements were conducted to characterize the overall drop size distribution with the cylinder removed. These tests were conducted in the $x z$-plane at $304.8 \mathrm{~mm}$ from the nozzle, representing the same plane as the front face of the cylinder. Figure 5 provides the cumulative 
drop size distribution results, demonstrating that the electrostatic charge had very little effect on the overall drop size distribution of the spray plume; Table 1 provides the relevant statics. Of note, the range of drop sizes is very similar spanning the range of approximately $25-550$ $\mu \mathrm{m}$, and the $D_{30}$ result is approximately $8 \%$ larger with $\mathrm{ES}_{\mathrm{on}}$. The fact that the $\mathrm{ES}_{\text {on }}$ produces a smaller $D_{V 0.99}$ result while also a larger $D_{30}$ indicates that the $\mathrm{ES}_{\text {on }}$ majority of droplets are increased in size slightly (say $8 \%$ ) while the rare largest droplets are slightly reduced in size. All laser diffraction tests were verified with three independent tests; each demonstrated an equivalent result.

Figure 6 shows the 2D spray distribution in $x z$-plane at $152.4 \mathrm{~mm}$ from the nozzle. Clearly the spray is concentrated in the center of the plume, and extends outward with a slightly elongated distribution on the lower-side fo the plume, due to gravitational forces acting on the droplets.

\begin{tabular}{|l|ccccccc|}
\hline Charge & $D_{\min }$ & $D_{10}$ & $D_{30}$ & $D_{V 0.1}$ & $D_{V 0.5}$ & $D_{V 0.9}$ & $D_{V 0.99}$ \\
\hline \hline ES $_{\text {off }}$ & 26 & 85 & 108 & 92 & 145 & 256 & 531 \\
SS $_{\text {on }}$ & 26 & 94 & 117 & 97 & 154 & 260 & 496 \\
\hline
\end{tabular}

Table 1. Drop size statistics at $304.8 \mathrm{~mm}$ spray distance with no cylinder in place. All values provided in $(\mu \mathrm{m})$.

\section{Spray Specific Charge}

The spray specific charge represents the potential for how well a spray plume of charged droplets could coat surfaces that are not directly in the path for direct impingement. Droplets carrying excess charge from free electrons or ions exude a repulsive electrostatic force on each other and an attractive electrostatic force on a grounded or neutral surface. Spray current measurements were acquired and resulted in an unsteady measurement between $0.3-0.5 \mu \mathrm{A}$ while spraying PathoCide $\AA$ at $360 \mathrm{ml} / \mathrm{min}$ with the Ryobi electrostatic sprayer. Using an average spray current of $0.4 \mu \mathrm{A}$, a spray specific charge of $67 \mu \mathrm{C} / \mathrm{kg}\left(0.067 \mathrm{C} / \mathrm{m}^{3}\right)$ was achieved. This spray specific charge is not large enough to significantly affect the atomization of the sprayed solution but is large enough to result in some coating on the back side of the cylinders sprayed for this investigation. When the electrostatic function was turned off for the Ryobi sprayer, a negligible spray current of $0.001 \mu \mathrm{A}$ was detected.

With PathoCide being a dilute hypochlorous acid solution in water, this spray specific charge measurement is of the same order of magnitude of the results by Marchewicz et al. [15] for water. It is important to note that Marchewicz charged their water spray using a high-voltage induction ring around the spray plume outside of the nozzle and had a larger flow rate of water than that tested for this experiment. The Ryobi electrostatic system used for this experiment uses a positive polarity high-voltage induction ring around the tube of liquid feeding the nozzle tip and it is not known to the authors what voltage is applied to the induction ring inside of the Ryobi sprayer. However, the measured spray specific charge sufficiently characterizes the transferred charge, and the mechanisms used to induce the charge become unimportant.

The varying spray current measured when spraying PathoCide with the Ryobi sprayer was not expected by the authors. To determine if this was a result of the PathoCide solution or the sprayer itself, a test was conducted to measure the spray current from deionized water sprayed with the Ryobi sprayer. This test produced a steady spray current of $0.55 \mu \mathrm{A}$ or a spray specific charge of $92 \mu \mathrm{C} / \mathrm{kg}\left(0.092 \mathrm{C} / \mathrm{m}^{3}\right)$. The steady spray current measurement with deionized water shows that the Ryobi sprayer provides a steady high-voltage to the induction ring. There are other factors resulting in the varying spray current with the PathoCide formulation, which could potentially be the result of induced chemical reactions like electrolysis of the water or decomposition of the hypochlorous acid. Further analysis and investigation falls outside of the scope of the present work and expertise of the authors and wasn't pursued further. 


\section{Summary and Conclusions}

An investigation of the coverage on a vertical cylinder using a disinfectant spray was evaluated without and with electrostatic charging of the spray droplets to explore the coverage improvement with charged droplets. Evaluation of the coverage on the cylinder was conducted using water sensitive paper and free available chlorine strips. It was found that charged droplets improved the overall coverage by $40 \%$ compared to uncharged droplets. Detailed analysis of the spray upstream of the cylinder was conducted examining the drop size distributon and spray pattern distribution; minimal differences were found between the charged and uncharged sprays with the charge spray providing an $8 \%$ increase in drop size $\left(D_{30}\right)$. Electrostatic charging of the droplets significantly improved the cylinder coverage with the disinfectant, and future efforts to examine the downstream drop size and velocity characteristics of the spray plume will be conducted.

\section{Acknowledgements}

The authors offer sincere thanks to Krunal Patel, Robert Till, and Kelvin Proano of Spraying Systems Co. for their contributions in data collection in support of these investigations.

\section{References}

[1] Law, S. E., "Electrostatic Pesticide Spraying: Concepts and Practice", IEEE Transactions on Industry Applications, vol. IA-19, no. 2, pp. 160-168, March 1983, doi: 10.1109/TIA.1983.4504176.

[2] Vesely, P.W., Schick, R.J., Shrimpton, J.S., and Mashayek, F., Energy efficient primary atomization of viscous food oils using an electrostatic method, Journal of Food Engineering, Vol. 237, pp. 27-32, 2018.

[3] Al-Ahmad, G., Shrimpton, J.S., Ergene, E.L., and Mashayek, F., Electrical performance of a charge-injection atomizer using viscous organic oils, Atomization and Sprays, Vol. 19(6), pp. 547-566, 2009.

[4] Shrimpton, J.S. and Yule, A.J., Characterisation of charged hydrocarbon sprays for application in combustion systems, Experiments in Fluids, Vol. 26(5), pp. 460-469, 1999.

[5] John Hopkins University of Medicine Coronavirus Resource Center, https:// coronavirus.jhu.edu/, Accessed: 2021-03-15

[6] Hung, L.S., Yao, S.C., "Experimental investigation of the impaction of water droplets on cylindrical objects", International Journal of Multiphase Flow, 25(8), pp. 1545-1559, 1999.

[7] Wang, Y., Wang, Y., Wang, S., "Droplet impact on cylindrical surfaces: Effects of surface wettability, initial impact velocity, and cylinder size", Journal of Colloid and Interface Science, 578, pp. 207-217, 2020.

[8] Lorenceau, E., Clanet, C., Quere, D., "Capturing drops with a thin fiber", Journal of Colloid and Interface Science, 279(1), pp. 192-197, 2004.

[9] Cantwell, B., Coles, D., "An experimental study of entrainment and transport in the turbulent near wake of a circular cylinder", J. Fluid Mech., 136, pp. 321 - 374, 1983.

[10] Perry A. E., Steiner, T. R., "Large-scale vortex structures in turbulent wakes behind bluff bodies. Part 1. Vortex formation processes", J. Fluid Mech., 174, 233-270, 1987.

[11] Keithley datasheet for model 6485 picoammeter, Keithley.

[12] Yule, A.J., Shrimpton, J.S., Watkins, A.P., Balachandran, W., and Hu, D., "Electrostatically atomized hydrocarbon sprays", Fuel, Vol. 74, No. 7, pp. 1094-1103, 1995.

[13] Vesely, P.J., Shrimpton, J.S., Mashayek, F., Schick, R. J., and Thenin, M., "Spray analysis of a multi-Orifice electrostatic atomization nozzle with high viscosity vegetable oils", ICLASS - 13th Triennial International Conference on Liquid Atomization and Spray Systems, Tainan, Taiwan, August 2015.

[14] Hoffmann, W.C., Hewitt, A.J., "Comparison of three imaging systems for water-sensitive papers", Applied Engineering in Agriculture, Technical Note, Vol. 21(6), pp. 961-964, 2005.

[15] Marchewicz, A., Soczyk, A.T., Krupa, A., and Jaworek, Electrostatic charging of water spray by induction, Journal of Physics: Conference Series, Vol. 1322, 2019. 